\section{The naked chromosome}

SpoIIIE and FtsK are bacterial DNA translocases involved in cell division. SpoIIIE has a key role in sporulating Bacillus subtilis, pumping around $70 \%$ of the chromosome from the mother-cell compartment into the forespore, across the asymmetric septum formed after DNA replication. Previous structural work on FtsK revealed a hexameric ring with a $30-\AA$ pore, large enough to accommodate DNA. Whether proteins bound to DNA can also go through this channel has now been investigated by Rudner and colleagues. The authors observed that RNA polymerase and DNA packaging and remodeling proteins $\mathrm{Hbs}$ and RacA are retained in the mother-cell compartment after DNA translocation. A similar observation was made for green fluorescent protein fused to TetR, targeted to an integrated array of tet $O$ sequences. Two fluorescent foci were initially seen in the mother cell, corresponding to the loci on each sister chromosome. When DNA translocation was completed, only one focus remained in the mother cell, with no fluorescence in the forespore. These in vivo data indicate that proteins are removed from DNA during translocation into the forespore. The recombinant motor domain of SpoIIIE could displace a stalled RNA polymerase complex assembled on DNA in vitro, in an ATP-dependent manner, demonstrating that the translocase activity of SpoIIIE can indeed remove proteins from DNA. The biological consequence of such stripping is that forespores receive their DNA in a 'reset' state, devoid of epigenetic marks and transcription factors, and ready to be reprogrammed. In fact, the authors observe a reduction in vegetative transcription in the forespore, probably owing to the removal

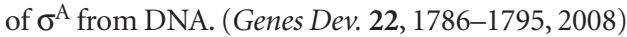

\section{IC}

\section{The core of the clock}

Circadian rhythms provide internal daily periodicity and are used by many organisms to anticipate daily changes in the environment. The molecular mechanism of these rhythms relies on oscillations at transcriptional, post-transcriptional and post-translational levels. In flies and mammals, the transcriptional cycles make up the core of the circadian feedback loop. A conserved heterodimeric transcription factor, made up of the proteins CLOCK and Bmal1 (CLK-BMAL) in mammals and Clock and Cycle (CLK-CYC) in flies, turns on the transcription of two repressor proteins, Timeless (TIM) and Period (PER) in flies, or Cryptochrome (CRY) and Period in mammals. These repressor proteins accumulate over the course of many hours and ultimately repress CLK-CYC or CLK-BMAL activity. These transcription-translation feedback loops produce the 24 -h rhythms in flies and mammals. However, recent findings in cyanobacteria suggest that post-translational modification of clock proteins is the central circadian mechanism. To directly test the effect of transcription on core circadian timekeeping in flies, Rosbash and colleagues generated a fusion protein between the CYC protein and the strong viral transcriptional activator VP16. This resulted in a CLK-CYC-VP16 complex with enhanced activity relative to that of the normal CLK-CYC complex. When expressed in S2 cells and flies, increased transcription was observed with reporter genes and the mRNA levels of direct CLK targets, including per and tim, were also increased. They also observed a shorter period in the $c y c-v p 16$ flies. Together, these results suggest that the main mechanism of circadian rhythm in flies involves transcription feedback. (PLoS Biol. 6, e119)

$B K$

\section{To find the right target}

Gene therapy holds the possibility of curing genetic diseases for which the gene (and responsible mutations) are known. In these cases, a wildtype copy of the gene in question would be integrated into somatic cells of a patient (knock in). Integration into a specific locus (gene targeting) would be preferred to random integration, to avoid the risk of disrupting DNA regions important for cellular functions. Gene targeting occurs via homologous recombination, a pathway for the repair of DNA double-stranded breaks (DSBs). However, a different DSB repair pathway, nonhomologous end-joining (NHEJ), usually predominates in somatic mammalian cells, resulting in integration into a random locus. The initial step in NHEJ is the binding of the heterodimeric complex Ku70-Ku86 to the ends of a DNA fragment. Now Hendrickson and colleagues find that reducing the levels of Ku70 in human cells in culture (by knocking out one copy of Ku70 (also known as XRCC6) and/or by knocking down its expression using small interfering RNAs or short hairpin RNAs) can increase the frequency of correct gene targeting up to 30 -fold. The authors used recombinant adeno-associated virus (rAAV) to target three different loci, with consistently higher targeting frequencies when Ku70 was depleted. rAAV is a promising vector for gene therapy, as it is nonpathogenic and can infect nondividing cells, and is currently undergoing clinical trials. Although the exact mechanisms by which depletion of Ku70 (and thus NHEJ) increases correct gene targeting in human cells are still under discussion, the implications of these findings for improving the efficiency of gene therapy are clear. (Proc. Natl. Acad. Sci. USA 105, 8703-8708, 2008)

\section{Separation switch}

The onset of chromosome separation during mitosis is a strikingly abrupt transition. Molecular components involved in anaphase have been identified and they include the anaphasepromoting complex (APC), a ubiquitin ligase that targets securin for destruction. Since securin is an inhibitor of separase, a protease that promotes separation, securin destruction promotes anaphase. Work from Morgan and colleagues now indicates that the protein kinase Cdk1 phosphorylates securin at residues near its ubiquitination site, preventing its ubiquitination. Conversely, dephosphorylation by the phosphatase Cdc 14 exposes securin to ubiquitination and turnover. As Cdc14 is in turn activated by separase, this suggests a positive feedback loop reinforcing securin destruction in a switch-like fashion, a property supported using mathematical modeling. The authors show that APCmediated securin ubiquitination is decreased upon in vitro phosphorylation by Cdk1, as predicted by the above model, and that $\mathrm{Cdc} 14$ reverses this effect. Using securin mutants lacking the phosphorylation sites, as well as temperature-sensitive separase and cdc14 mutants, the authors monitored separation of chromosomes IV and $\mathrm{V}$ in yeast, finding a less abrupt anaphase in the mutant backgrounds, with segregation occurring over a longer time frame. Other defects observed in these securin mutants included problems in mitotic spindle behavior, perhaps because the abrupt degradation of securin normally ensures that sister separation is coordinated with dephosphorylation of factors regulating the spindle. Interestingly, the authors note that chromosomes IV and V consistently separate in the same order, and further work will test whether different separase activation thresholds trigger segregation of distinct chromosomes as proposed. (Nature, advance online publication 15 June 2008 doi:10.1038/nature07050) 